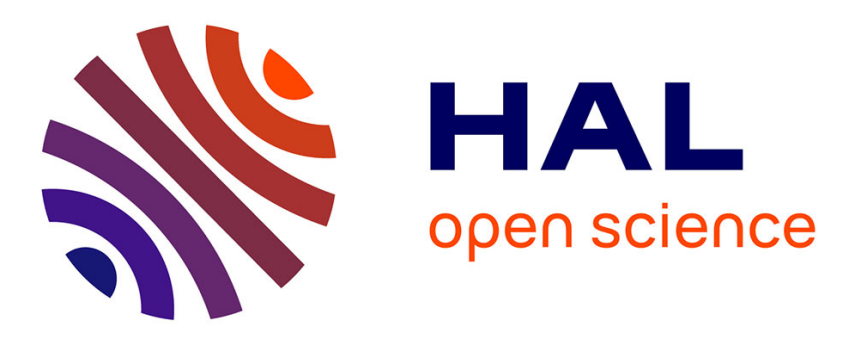

\title{
Sparsity-based localization of spatially coherent distributed sources
}

Wenmeng Xiong, José Picheral, Sylvie Marcos, Gilles Chardon

\section{To cite this version:}

Wenmeng Xiong, José Picheral, Sylvie Marcos, Gilles Chardon. Sparsity-based localization of spatially coherent distributed sources. IEEE International Conference on Acoustics, Speech and Signal Processing (ICASSP), Mar 2016, Shanghai, China. 10.1109/ICASSP.2016.7472276 hal-01231789

\section{HAL Id: hal-01231789 \\ https://hal.science/hal-01231789}

Submitted on 20 Nov 2015

HAL is a multi-disciplinary open access archive for the deposit and dissemination of scientific research documents, whether they are published or not. The documents may come from teaching and research institutions in France or abroad, or from public or private research centers.
L'archive ouverte pluridisciplinaire HAL, est destinée au dépôt et à la diffusion de documents scientifiques de niveau recherche, publiés ou non, émanant des établissements d'enseignement et de recherche français ou étrangers, des laboratoires publics ou privés. 


\title{
PERFORMANCE ANALYSIS OF MUSIC IN THE PRESENCE OF MODELING ERRORS DUE TO THE SPATIAL DISTRIBUTIONS OF SOURCES
}

\author{
Wenmeng XIONG José PICHERAL Sylvie MARCOS \\ Lss,CNRS,Paris-sud,CentraleSupelec,91192 GIF-sur-Yvette,France \\ firstname.lastname@12s.centralesupelec.fr
}

\begin{abstract}
In this paper, the direction of arrival (DOA) localization of spatially distributed sources impinging on a sensor array is considered. The performance of the well known MUSIC estimator is studied in the presence of model errors due to angular dispersion of sources. Taking into account the coherently distributed source model proposed in [1], we establish closed-form expressions of the DOA estimation error and mean square error (MSE) due to both the model errors and the effects of a finite number of snapshots. The analytical results are validated by numerical simulations and allow to analyze the performance of MUSIC for coherently distributed sources.
\end{abstract}

Index Terms - array signal processing, distributed sources, model error, performance, MUSIC

\section{INTRODUCTION}

The effects of model errors on DOA estimation has been widely studied in the literature with the sources assumed to be far-field point transmitters or reflectors [2]. In many applications, for instance, aero-acoustic sources imaging [3], ocean waves parameter estimation [4], bio-medical source localization [5] or also mobile communication [6], the physical sources can no longer be considered as point sources and a spatially distributed model of the sources could be more appropriate.

The models for spatially distributed sources have been classified into two types, namely incoherently distributed (ID) sources and coherently distributed (CD) sources. On one hand, for ID sources, signals coming from different points of the same distributed source can be considered uncorrelated [7] [8]. On the other hand, in the scenario of CD sources, the received signal components are delayed and scaled replicas from different points of the same one [9] [10].

In the literature, different techniques propose to estimate the nominal DOA of distributed sources but they generally require that the shape of the dispersion is known which may not be true in practice. Even if it is the case, a mismatch on the angular spread dispersion between the real signals and the models will bring estimation errors on the DOA estimates.

In previous works, the model errors have been considered, in the case of punctual sources, as uncertainties on the array elements positions or patterns, or inter-element coupling, and are described as random variables [11] [12] [13] [14]. For ID sources, the performance analyses have been investigated in the presence of the model error as a mismatch between the angular dispersion of the actual sources and the MUSIC estimator in [15], and the ultimate asymptotic performance, that is to say, the lower bound of the estimation covariance has been investigated in [16]. We here focus on the CD sources model proposed in [1], which is well adapted to the applications such as the aero-acoustic sources imaging [3]. The MUSIC estimator has been extended for a model with an angular dispersion, and the model error refers to a mismatch between the angular dispersion of the actual sources and that of the extendedMUSIC estimator in our scenario.

In this paper, we first propose a new approach to obtain an accurate approximated analytical expression of the DOA estimation error and MSE. Then, the finite sample effects due to the estimation of the received data covariance matrix will be investigated. Finally, assuming that the shape of the angular distribution is known, an analytical expression of the DOA estimation error as a polynomial function of the angular spread dispersion error is proposed. This expression allows to see explicitly the influence of the model error on the DOA estimation performance, and could be useful in a future work to optimize the antenna parameters in order to reduce the DOA estimation error due to the angular spread of the sources.

The organization of this paper is as follows. The signal model and $1 D$-DSPE are given in section 2 . In section 3 , the sensitivity of the estimator is theoretically analyzed. Numerical simulations are presented in section 4 to validate the analytical expressions of the previous section. Finally, conclusions are given is section 5 .

\section{SIGNAL MODEL AND $1 D$ ESTIMATOR FOR DISTRIBUTED SOURCES}

Let us consider $q$ spatially CD far-field sources impinging on an array of $M$ sensors. The $q$ sources and the $M$ signals received at the array at moment $t$ are denoted by $\mathbf{s}(t)=\left[s_{1}(t), \ldots, s_{q}(t)\right]^{T}$ and $\mathbf{y}(t)=\left[y_{1}(t), \ldots, y_{M}(t)\right]^{T}$, respectively, with:

$$
\mathbf{y}(t)=\mathbf{C}(\theta) \mathbf{s}(t)+\mathbf{n}(t)
$$

where $\mathbf{n}(t) \in \mathbb{C}^{M \times 1}$ represents the complex Gaussian distributed additive noise, $\mathbf{C}(\theta)=\left[\mathbf{c}_{h_{1}}\left(\theta_{1}\right), \ldots, \mathbf{c}_{h_{q}}\left(\theta_{q}\right)\right] \in \mathbb{C}^{M \times q}$ is the array steering matrix composed of $q$ steering vectors $\mathbf{c}_{h i}(\theta)$ that can be written as proposed in [1] by:

$$
\mathbf{c}_{h i}(\theta)=\int_{-\frac{\pi}{2}}^{\frac{\pi}{2}} \mathbf{a}(\theta+\phi) h_{i}(\phi) d \phi,
$$

where $i=1 \ldots q$, and $\mathbf{a}(\theta)$ is the steering vector for a point source which arrives from the DOA $\theta$. In the most general case, the steering vector $\mathbf{a}(\theta)$ is also a function of the array geometry, the sensor gains, the form of the wavefront, and other possible parameters which are supposed to be known. The function $h(\phi)$ is introduced 
to describe the angular spread distribution (for instance, Uniform and Gaussian distributions) and it can be parameterized by an angular dispersion $\Delta$ which is omitted in the notation. The source signals and the additive noise are considered to be centered Gaussian independent random variables. Assuming that signals and noises are uncorrelated and the sources are uncorrelated with each other, the correlation matrix is given by:

$$
\mathbf{R}_{y}=E\left[\mathbf{y} \mathbf{y}^{H}\right]=\mathbf{C R}_{s} \mathbf{C}^{H}+\sigma_{b}^{2} \mathbf{I},
$$

where $E[$.$] is the expectation operator, \mathbf{R}_{s}$ and $\sigma_{b}^{2}$ are the source covariance matrix and the noise variance, respectively.

The DSPE method proposed in [1] is a $2 D$ MUSIC-like estimator allowing to jointly estimate the two parameters $\theta$ and $\Delta$ by maximizing the criterion $\frac{1}{\left\|\mathbf{c}_{h}^{H}(\theta, \Delta) \mathbf{V}_{b}\right\|^{2}}$, where $\mathbf{V}_{b}$ is the matrix of the $M-q$ eigenvectors associated to the smallest eigenvalue of $\mathbf{R}_{y}$ and which spans the noise subspace. However, the form of $h(\phi)$ may not be known in practice. In the following, we will consider that the shape of $h(\phi)$ and $\Delta$ are imperfectly known and that the only parameter to estimate is $\theta$. We will then investigate the effect of an imperfect knowledge of $h(\phi)$ and $\Delta$, it is to say of $\mathbf{c}_{h}(\phi)$, on the $1 D$-DSPE algorithm therefore denoted by:

$$
\hat{\theta}_{i}=\arg \max _{\theta} \frac{1}{\left\|\mathbf{c}_{h}^{H}(\theta) \mathbf{V}_{b}\right\|^{2}}
$$

\section{PERFORMANCE ANALYSIS}

\subsection{General case}

The data covariance matrix $\mathbf{R}_{y 0}=E\left[\mathbf{y}_{0} \mathbf{y}_{0}^{H}\right]$ is estimated from $N$ snapshots $\left\{\mathbf{y}_{0}\left(t_{n}\right)\right\}_{n=1, \ldots, N}$ by $\hat{\mathbf{R}}_{y 0} \approx \frac{1}{N} \sum_{n=1}^{N} \mathbf{y}_{0}\left(t_{n}\right) \mathbf{y}_{0}^{H}\left(t_{n}\right)$. It follows that the DOA estimator of $\theta_{i}$ is :

$$
\hat{\theta}_{i}=\arg \max _{\theta} \frac{1}{\left\|\mathbf{c}_{h}^{H}(\theta) \hat{\mathbf{V}}_{b 0}\right\|^{2}},
$$

where $\hat{\mathbf{V}}_{b 0}$ is associated to the eigendecomposition of:

$$
\hat{\mathbf{R}}_{y 0}=\hat{\mathbf{V}}_{s 0} \hat{\Lambda}_{s 0} \hat{\mathbf{V}}_{s 0}^{H}+\hat{\mathbf{V}}_{b 0} \hat{\Lambda}_{b 0} \hat{\mathbf{V}}_{b 0}^{H},
$$

and $\mathbf{c}_{h}(\theta)$ is given by (2) but with a form of the angular spread distribution $h(\phi)$ which may be different from the true distribution of the actual sources $h_{0 i}(\phi)$. Note that the parameters related to the true values are indexed by 0 to distinguish them from parameters used by the estimator. The theoretical covariance matrix of the signal related to the actual steering matrix $\mathbf{C}_{h 0}(\theta)$ is thus defined as $\mathbf{R}_{y 0}=\mathbf{V}_{s 0} \Lambda_{s 0} \mathbf{V}_{s 0}^{H}+\sigma_{b}^{2} \mathbf{V}_{b 0} \mathbf{V}_{b 0}^{H}$, and we write $\mathbf{R}_{y 0}=\mathbf{R}_{y h}+\Delta \mathbf{R}_{y h}$, where $\mathbf{R}_{y h}$ would correspond to the covariance matrix of the signal related to a steering vector $\mathbf{c}_{h}(\theta)$ and where $\mathbf{R}_{y h}=\mathbf{V}_{s h} \Lambda_{s h} \mathbf{V}_{s h}^{H}+\sigma_{b}^{2} \mathbf{V}_{b h} \mathbf{V}_{b h}^{H}$.

We introduce the error on the steering vector model $\Delta \mathbf{c}=$ $\mathbf{c}_{h}\left(\theta_{i}\right)-\mathbf{c}_{h 0 i}\left(\theta_{i}\right)$. We also note $\Delta \mathbf{V}_{b h}=\mathbf{V}_{b 0}-\mathbf{V}_{b h}$ where $\Delta \mathbf{V}_{b h}$ is an error on the noise eigenmatrix due to the model mismatch between $\mathbf{c}_{h}$ and $\mathbf{c}_{h 0}$. Let us also introduce the error due to the finite number of snapshots $N$ on the estimation of the data covariance matrix $\Delta \mathbf{R}_{y 0}=\hat{\mathbf{R}}_{y 0}-\mathbf{R}_{y 0}$ and the error on the noise corresponding eigenmatrix $\Delta \mathbf{V}_{b 0}=\hat{\mathbf{V}}_{b 0}-\mathbf{V}_{b 0}$. The error due to the finite number of snapshots on the noise subspace projector is $\Delta \Pi_{b 0}=\hat{\mathbf{V}}_{b 0} \hat{\mathbf{V}}_{b 0}^{H}-\mathbf{V}_{b 0} \mathbf{V}_{b 0}^{H}$, with $\Pi_{b 0}=\mathbf{V}_{b 0} \mathbf{V}_{b 0}^{H}$. According to [2], the relation of $\Delta \Pi_{b 0}$ and $\Delta \mathbf{R}_{b 0}$ is:

$$
\Delta \Pi_{b 0}=-\Pi_{b 0} \Delta \mathbf{R}_{y 0} \mathbf{Q}-\mathbf{Q} \Delta \mathbf{R}_{y 0} \Pi_{b 0},
$$

where $\mathbf{Q}=\mathbf{V}_{s 0}\left(\Lambda_{s 0}-\sigma_{b}^{2} \mathbf{I}\right)^{-1} \mathbf{V}_{s 0}$, I is a $q \times q$ identity matrix.

According to (5), the DOA estimation $\hat{\theta}_{i}$ satisfies that the first order derivative of the denominator of (5) equals zero which yields:

$$
2 \mathcal{R} e\left\{\dot{\mathbf{c}}_{h}^{H}\left(\hat{\theta}_{i}\right) \hat{\mathbf{V}}_{b 0} \hat{\mathbf{V}}_{b 0}^{H} \mathbf{c}_{h}\left(\hat{\theta}_{i}\right)\right\}=0,
$$

where $\dot{\mathbf{c}}_{h}\left(\hat{\theta}_{i}\right)=\left.\frac{\partial \mathbf{c}(\theta)}{\partial \theta}\right|_{\hat{\theta}_{i}}$. Assuming that, $\hat{\theta}_{i}$ is not far from $\theta_{i}$, we introduce the second order Taylor series approximations of $\mathbf{c}_{h}(\theta)$ and $\dot{\mathbf{c}}_{h}(\theta)$ in (8), and exploiting (7) to substitute $\hat{\mathbf{V}}_{b 0} \hat{\mathbf{V}}_{b 0}$ yields:

$$
A\left(\theta_{i}\right) \Delta \theta_{i}^{2}+B\left(\theta_{i}\right) \Delta \theta_{i}+C\left(\theta_{i}\right)=0
$$

where the terms of order greater than 2 in $\Delta \theta_{i}$ have been neglected, and the scalar $A, B, C$ are defined with $B=B_{1}+B_{2}$ and $C=C_{1}+C_{2}$ :

$$
\begin{aligned}
A\left(\theta_{i}\right)= & \mathcal{R} e\left\{\frac{1}{2} \dot{\mathbf{c}}_{h}\left(\theta_{i}\right)^{H} \mathbf{V}_{b 0} \mathbf{V}_{b 0}^{H} \ddot{\mathbf{c}}_{h}\left(\theta_{i}\right)\right. \\
& \left.+\ddot{\mathbf{c}}_{h}\left(\theta_{i}\right)^{H} \mathbf{V}_{b 0} \mathbf{V}_{b 0}^{H} \dot{\mathbf{c}}_{h}\left(\theta_{i}\right)+\frac{1}{2} \dddot{\mathbf{c}}_{h}\left(\theta_{i}\right)^{H} \mathbf{V}_{b 0} \mathbf{V}_{b 0}^{H} \mathbf{c}_{h}\left(\theta_{i}\right)\right\}, \\
B_{1}\left(\theta_{i}\right)= & \mathcal{R} e\left\{\dot{\mathbf{c}}_{h}\left(\theta_{i}\right)^{H} \mathbf{V}_{b 0} \mathbf{V}_{b 0}^{H} \dot{\mathbf{c}}_{h}\left(\theta_{i}\right)+\ddot{\mathbf{c}}_{h}\left(\theta_{i}\right)^{H} \mathbf{V}_{b 0} \mathbf{V}_{b 0}^{H} \mathbf{c}_{h}\left(\theta_{i}\right)\right\},
\end{aligned}
$$

$$
\begin{aligned}
& E\left[\Delta \theta_{i}^{2}\right]=\frac{B_{1}\left(\theta_{i}\right)^{2}-2 A\left(\theta_{i}\right) C_{1}\left(\theta_{i}\right)-B_{1}\left(\theta_{i}\right) \sqrt{B_{1}\left(\theta_{i}\right)^{2}-4 A\left(\theta_{i}\right) C_{1}\left(\theta_{i}\right)}}{2 A\left(\theta_{i}\right)^{2}} \\
& +\frac{\sigma_{b}^{2}}{N}\left[\left(\frac{1}{4 A\left(\theta_{i}\right)^{2}}-\frac{B_{1}\left(\theta_{i}\right)}{4 A\left(\theta_{i}\right)^{2} \sqrt{B_{1}\left(\theta_{i}\right)^{2}-4 A\left(\theta_{i}\right) C_{1}\left(\theta_{i}\right)}}\right) \varphi\left(\theta_{i}\right)+\frac{\chi\left(\theta_{i}\right)}{2 A\left(\theta_{i}\right) \sqrt{B_{1}\left(\theta_{i}\right)^{2}-4 A\left(\theta_{i}\right) C_{1}\left(\theta_{i}\right)}}\right],
\end{aligned}
$$

where:

$$
\begin{aligned}
\varphi\left(\theta_{i}\right)= & \mathcal{R} e\left\{4 \dot{\mathbf{c}}\left(\theta_{i}\right)^{H} \mathbf{V}_{b 0} \mathbf{V}_{b 0}^{H} \dot{\mathbf{c}}\left(\theta_{i}\right) \dot{\mathbf{c}}\left(\theta_{i}\right)^{H} \mathbf{Q} \mathbf{R}_{y 0} \mathbf{Q} \dot{\mathbf{c}}\left(\theta_{i}\right)+4 \ddot{\mathbf{c}}\left(\theta_{i}\right)^{H} \mathbf{V}_{b 0} \mathbf{V}_{b 0}^{H} \dot{\mathbf{c}}\left(\theta_{i}\right) \mathbf{c}\left(\theta_{i}\right) \mathbf{Q R} \mathbf{R}_{y 0} \mathbf{Q} \mathbf{c}\left(\theta_{i}\right)\right. \\
& \left.+\ddot{\mathbf{c}}\left(\theta_{i}\right)^{H} \mathbf{V}_{b 0} \mathbf{V}_{b 0}^{H} \ddot{\mathbf{c}}\left(\theta_{i}\right) \mathbf{c}\left(\theta_{i}\right) \mathbf{Q} \mathbf{R}_{y 0} \mathbf{Q} \mathbf{c}\left(\theta_{i}\right)\right\}, \\
\chi\left(\theta_{i}\right)= & \mathcal{R} e\left\{2 \dot{\mathbf{c}}\left(\theta_{i}\right)^{H} \mathbf{V}_{b 0} \mathbf{V}_{b 0}^{H} \dot{\mathbf{c}}\left(\theta_{i}\right) \dot{\mathbf{c}}\left(\theta_{i}\right) \mathbf{Q} \mathbf{R}_{y 0} \mathbf{Q} \mathbf{c}\left(\theta_{i}\right)+\ddot{\mathbf{c}}\left(\theta_{i}\right)^{H} \mathbf{V}_{b 0} \mathbf{V}_{b 0}^{H} \dot{\mathbf{c}}\left(\theta_{i}\right) \mathbf{c}\left(\theta_{i}\right) \mathbf{Q} \mathbf{R}_{y 0} \mathbf{Q} \mathbf{c}\left(\theta_{i}\right)\right\} .
\end{aligned}
$$




$$
\begin{aligned}
B_{2}\left(\theta_{i}\right)= & \mathcal{R} e\left\{2 \dot{\mathbf{c}}_{h}\left(\theta_{i}\right)^{H} \mathbf{V}_{b 0} \mathbf{V}_{b 0}^{H} \Delta \mathbf{R}_{y 0} \mathbf{Q} \dot{\mathbf{c}}_{h}\left(\theta_{i}\right)\right. \\
& \left.+\ddot{\mathbf{c}}_{h}\left(\theta_{i}\right)^{H} \mathbf{V}_{b 0} \mathbf{V}_{b 0}^{H} \Delta \mathbf{R}_{y 0} \mathbf{Q} \mathbf{c}_{h}\left(\theta_{i}\right)\right\}, \\
C_{1}\left(\theta_{i}\right)= & \mathcal{R} e\left\{\dot{\mathbf{c}}_{h}\left(\theta_{i}\right)^{H} \mathbf{V}_{b 0} \mathbf{V}_{b 0}^{H} \mathbf{c}_{h}\left(\theta_{i}\right)\right\}, \\
C_{2}\left(\theta_{i}\right)= & \mathcal{R} e\left\{\dot{\mathbf{c}}_{h}\left(\theta_{i}\right)^{H} \mathbf{V}_{b 0} \mathbf{V}_{b 0}^{H} \Delta \mathbf{R}_{y 0} \mathbf{Q} \mathbf{c}_{h}\left(\theta_{i}\right)\right\} .
\end{aligned}
$$

The expression of $\Delta \theta_{i}$ can be obtained by solving the $2^{\text {nd }}$ order equation (9), and taking into account the convexity of the criterion to select the right root, so that:

$$
\Delta \theta_{i}=\frac{-B\left(\theta_{i}\right)+\sqrt{B\left(\theta_{i}\right)^{2}-4 A\left(\theta_{i}\right) C\left(\theta_{i}\right)}}{2 A\left(\theta_{i}\right)} .
$$

In addition, according to the formulas in [14], the MSE can be expressed by (11), as shown at the bottom of the previous page, exploiting the fact that $B_{2}\left(\theta_{i}\right)$ and $C_{2}\left(\theta_{i}\right)$ are random terms related to $\Delta \mathbf{R}_{y 0}$, while $A\left(\theta_{i}\right), B_{1}\left(\theta_{i}\right), C_{1}\left(\theta_{i}\right)$ are deterministic, and using $E\left[\Delta \mathbf{R}_{y 0}\right]=0$.

It is interesting to see that the MSE is composed of two terms, one depends only on the model error, the other depends on the model error but with a factor $\sigma^{2} / N$. It can be expected that the second term will be negligible when $N$ increases.

\subsection{First order approximation}

In this subsection, we discuss the situation that the estimation error $\Delta \theta_{i}$ is small enough, so that the second order terms in $\Delta \theta_{i}$ can be negligible with respect to the first order terms. Keeping only the first order terms in (9), replacing $\mathbf{V}_{b 0}$ by $\mathbf{V}_{b h}+\Delta \mathbf{V}_{b h}$, neglecting the second order terms in $\Delta \mathbf{V}_{b 0} \Delta \theta_{i}$ and $\Delta \mathbf{V}_{b h} \Delta \theta_{i}$, and using $\mathbf{R}_{y h} \mathbf{V}_{b 0}=\mathbf{V}_{b 0} \Lambda_{b h}$, the estimation error can be simplified as:

$$
\begin{aligned}
\Delta \theta_{i} & =\frac{\mathcal{R} e\left\{\dot{\mathbf{c}}_{h}^{H}\left(\theta_{i}\right) \mathbf{V}_{b h} \mathbf{V}_{b h}^{H} \Delta \mathbf{c}\right\}}{\dot{\mathbf{c}}_{h}^{H}\left(\theta_{i}\right) \mathbf{V}_{b h} \mathbf{V}_{b h}^{H} \dot{\mathbf{c}}_{h}\left(\theta_{i}\right)} \\
& +\frac{\mathcal{R} e\left\{\dot{\mathbf{c}}_{h}^{H}\left(\theta_{i}\right) \mathbf{V}_{b h} \mathbf{V}_{b h}^{H} \Delta \mathbf{R}_{y 0} \mathbf{Q \mathbf { c } _ { h }}\left(\theta_{i}\right)\right\}}{\dot{\mathbf{c}}_{h}^{H}\left(\theta_{i}\right) \mathbf{V}_{b h} \mathbf{V}_{b h}^{H} \dot{\mathbf{c}}_{h}\left(\theta_{i}\right)} .
\end{aligned}
$$

The MSE can be given by:

$$
\begin{aligned}
& E\left[\Delta \theta_{i}^{2}\right]=\left(\frac{\mathcal{R} e\left\{\dot{\mathbf{c}}_{h}^{H}\left(\theta_{i}\right) \mathbf{V}_{b h} \mathbf{V}_{b h}^{H} \Delta \mathbf{c}\right\}}{\dot{\mathbf{c}}_{h}^{H}\left(\theta_{i}\right) \mathbf{V}_{b h} \mathbf{V}_{b h}^{H} \dot{\mathbf{c}}_{h}\left(\theta_{i}\right)}\right)^{2} \\
& +\frac{\sigma_{b}^{2}}{2 N} \cdot \frac{\mathcal{R} e\left\{\dot{\mathbf{c}}_{h}^{H}\left(\theta_{i}\right) \mathbf{V}_{b h} \mathbf{V}_{b h}^{H} \dot{\mathbf{c}}_{h}\left(\theta_{i}\right) \mathbf{c}_{h}^{H}\left(\theta_{i}\right) \mathbf{R}_{y 0} \mathbf{Q} \mathbf{c}_{h}\left(\theta_{i}\right)\right\}}{\left(\dot{\mathbf{c}}_{h}^{H}\left(\theta_{i}\right) \mathbf{V}_{b h} \mathbf{V}_{b h}^{H} \dot{\mathbf{c}}_{h}\left(\theta_{i}\right)\right)^{2}} .
\end{aligned}
$$

As already noted for (11), the second term decreases when $N$ increases, which is not the case for the first terms of (13) and (14). Note that this simplification allows to express the first terms of (13) and (14) as a function of the model error $\Delta \mathbf{c}$.

\subsection{Estimation error as an explicit function of the model error}

In this subsection, we assume that the covariance matrix is perfectly known and we only study the deterministic terms of (10) so that $B_{2}$ and $C_{2}$ are null. We assume that in this case, the shape of $h$ related to the actual signals sources is known, where all the sources have the same shape and the same angular spread dispersion $\Delta_{0}$. The model error is therefore caused by the error on $\Delta$ or by the fact that the sources are assumed to be points when the classical MUSIC estimator is used. Assuming again that $\Delta$ is not far from $\Delta_{0}$, and noting $\delta=\Delta-\Delta_{0}$, we can introduce the second order Taylor series approximations in $\delta$ :

$$
\mathbf{c}_{h}\left(\theta_{i}\right) \approx \mathbf{c}_{h 0}\left(\theta_{i}\right)+\delta \mathbf{g}_{h 01}\left(\theta_{i}\right)+\frac{1}{2} \delta^{2} \mathbf{g}_{h 02}\left(\theta_{i}\right),
$$

where $\mathbf{g}_{h 01}\left(\theta_{i}\right)=\left.\frac{\partial \mathbf{c}_{h 0}\left(\theta_{i}\right)}{\partial \Delta}\right|_{\Delta_{0}}, \mathbf{g}_{h 02}\left(\theta_{i}\right)=\left.\frac{\partial^{2} \mathbf{c}_{h 0}\left(\theta_{i}\right)}{\partial \Delta^{2}}\right|_{\Delta_{0}}$. We can pay attention that $\mathbf{g}_{h 01}$ and $\mathbf{g}_{h 02}$ reveals the sensibility of our model to the variation of the angular dispersion of the actual signal. The estimation error can be thus given by:

$$
\Delta \theta_{i}=\frac{-\left(f_{4}+f_{5} \delta+f_{6} \delta^{2}\right)+\sqrt{x+y \delta+z \delta^{2}+w \delta^{3}+k \delta^{4}}}{2\left(f_{1}+f_{2} \delta+f_{3} \delta^{2}\right)},
$$

where $f_{i}(i=1, \ldots, 5)$ and $x, \ldots, k$ can be expressed in function of $\mathbf{V}_{b 0}$, derivatives of $\mathbf{g}$ and $\mathbf{c}$. Details can be found in [17].

Ignoring the second terms in $\Delta \theta_{i}$ in (9) and extending Taylor series in $\delta$ to third order terms for an accurate approximation, the estimation error can be further expressed as a polynomial in $\delta$ :

$$
\Delta \theta_{i}=\alpha\left(\theta_{i}, \Delta_{0}\right) \delta+\beta\left(\theta_{i}, \Delta_{0}\right) \delta^{2}+\gamma\left(\theta_{i}, \Delta_{0}\right) \delta^{3},
$$

where $\alpha, \beta$ and $\gamma$ are functions depending on the source signals and sensor parameters (see [17] for details).

\section{NUMERICAL SIMULATIONS}

In this section, numerical examples are presented to illustrate the validity of the analytical results of the estimation performances established in section 3 . In all simulations, a uniform linear array composed of $M=10$ sensors spaced by $d=\lambda / 2$ is considered, $S N R=10 d B$, and $N=1000$ snapshots.

Figure 1 exhibits the estimation error or bias on $\theta$ for two Uniform distributed sources at $\theta_{1}=21^{\circ}$ and $\theta_{2}=39^{\circ}$, respectively, with an angular dispersion $\Delta_{0}=10^{\circ}$. The model error due to the dispersion parameter $\Delta$ has been varied to study its effect on the DOA's estimation accuracy. As expected the bias is null when the exact model parameter $\Delta=\Delta_{0}=10^{\circ}$ is used. We can observe that the estimation error is smaller when the parameter $\Delta$ is smaller than $\Delta_{0}$, than when it is larger than $\Delta_{0}$. Focusing on the validity of the expression derived in the previous section, we can notice that for $\theta_{2}$, the DOA estimation error $\Delta \theta_{2}$ obtained in (10) and the one obtained in (13) both match the simulation results. However, for $\theta_{1}$, which has an estimation error much bigger than $\theta_{2}$ when the model error increases, the result obtained in (10) with the second order approximation outperforms the one in (13) with the first order approximation.

In Figure 2, the RMSE of $\theta$ evaluated with 100 Monte Carlo simulations is plotted versus the snapshot number, with the model parameter set to $\Delta=15^{\circ}$. The MSE decreases as well as $N$ increases, and then converges to a non zero value whose expression is given in (11). This reveals that when there are two perturbations, the finite number of snapshots effect dominates in the case of a small number of snapshots whereas the model error effect dominates when the snapshots number is large. Again we can see that as in Figure 1, the result with the second order approximation outperforms the one with the first order approximation.

Figure 3 illustrates the approximation obtained in (16) and (17), in order to express the estimation error as an explicit function of the model error, in the same previous scenario. We can observe that, in this case, (16) fits better the simulation results, while 


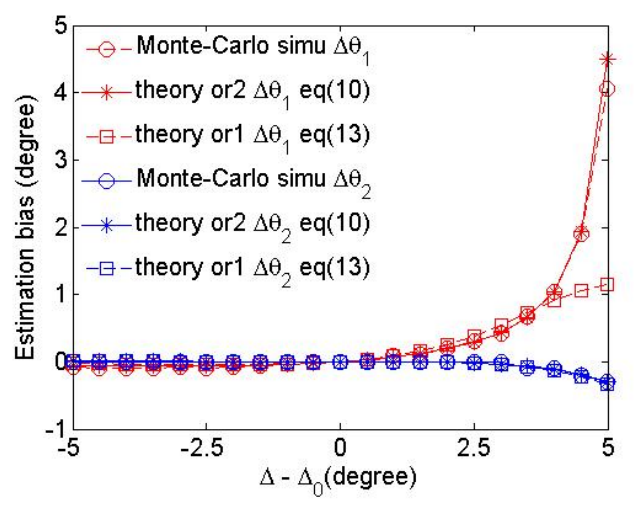

Figure 1: DOA estimation error vs. angular dispersion model error ( 2 sources with Uniform angular dispersion, $\Delta_{0}=10^{\circ}, \theta_{1}=$ $21^{\circ}, \theta_{2}=39^{\circ}$ )

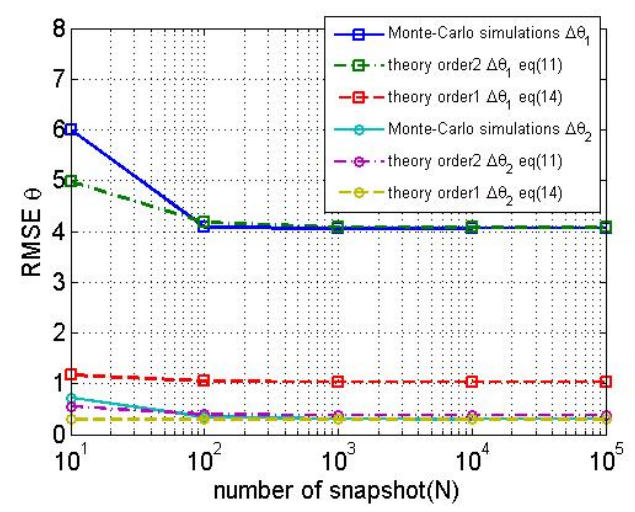

Figure 2: RMSE of DOA estimation error of $\theta_{1}$ and $\theta_{2}$ vs. number of snapshots $(\mathrm{N})$ (2 sources with Uniform angular dispersion, $\Delta_{0}=10^{\circ}, \Delta=15^{\circ}, \theta_{1}=21^{\circ}, \theta_{2}=39^{\circ}$ )

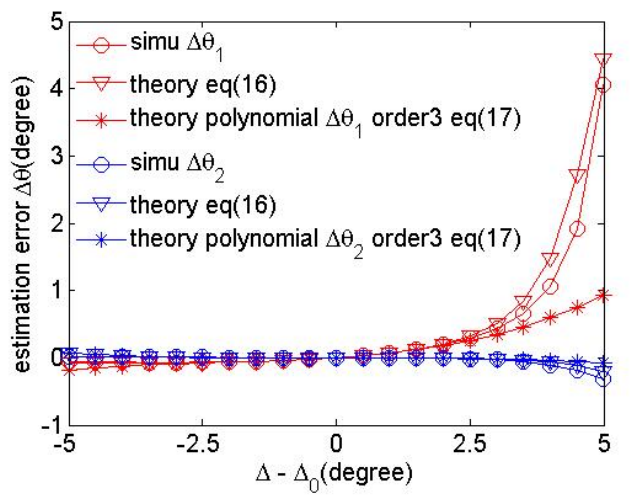

Figure 3: DOA estimation error vs. angular dispersion model error ( 2 sources with Uniform angular dispersion, $\Delta_{0}=10^{\circ}, \theta_{1}=$ $21^{\circ}, \theta_{2}=39^{\circ}$ )

the polynomial expression (17) fits the simulation results only for small model errors.
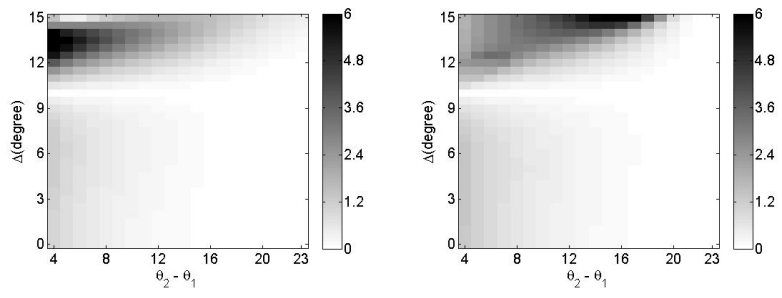

(a) Theory with order 1 approxi-(b) Theory with order 2 approximation in (10) mation in (13)

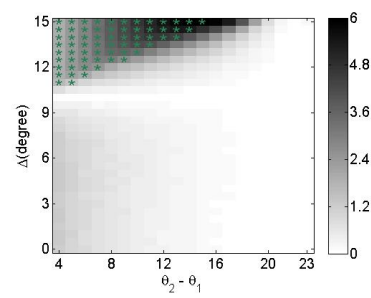

(c) Simulation

Figure 4: Absolute value of estimation error $\left|\Delta \theta_{1}\right|$ vs. source angular separation $\left|\theta_{2}-\theta_{1}\right|$ and model angular dispersion $\Delta(2$ sources with Uniform angular dispersion, $\Delta_{0}=10^{\circ}, 100$ MonteCarlo simulations)

Figure 4 shows the results of the absolute value of the DOA estimation error when the model error due to the dispersion parameter and the angle between the two sources both vary, with $\theta_{m}=\frac{1}{2}\left(\theta_{1}+\theta_{2}\right)=30^{\circ}$. The green stars mark the region where the sources are not resolved, that is to say, the two sources are so close that the criterion $1 D$-DSPE gives the false appearance that there is only one source in the middle. These results allow to highlight two behaviors of the estimators: firstly, the closer the sources are, the more the model error impacts on the estimation accuracy; secondly, when the model error is small enough, high resolution is achieved. Taking into account that a distributed estimator with $\Delta=0^{\circ}$ means the classical estimator MUSIC with a point source model, and $\Delta=10^{\circ}$ is the 1D-DSPE without model error, the advantage of a distributed estimator with respect to a classical MUSIC is highlighted. In addition to the fact that the theoretical results correspond to the simulation results, we can observe that again the result obtained in (10) works better than the result obtained in (13), even in the case where there are resolution problems.

\section{CONCLUSION}

In this paper, we have investigated the effects of both the angular dispersion of the source and the finite number of snapshots on the behavior of the DOA MUSIC-based estimator. New analytical expressions of the DOA estimation error and MSE as a function of these two perturbations have been given. Particularly, in the special case when the theoretical covariance matrix is available, expressions as an explicit function of the model error is proposed, which gives an easier way to analyze the influence of a model error, or to optimize the array configurations to reduce the DOA estimation error. Simulations which are carried out are in adequacy with the proposed theoretical results. The performance of MUSIC for coherently distributed sources can thus be analyzed. 


\section{REFERENCES}

[1] S.Valaee, B.Champagne, and P.Kabal, "Parametric localization of distributed sources," IEEE Transactions on Signal Processing, vol. 43, no. 9, pp. 2144-2153, Sept. 1995.

[2] A.J.Weiss and B.Friedlander, "Effects od modeling errors on the resolution threshold of the MUSIC algorithm," IEEE Transactions on Signal Processing, vol. 42, no. 6, pp. 15191526, June 1994.

[3] N. Chu, A. M. Djafari, and J. Picheral, "Robust Bayesian super-resolution approach via sparsity enforcing a priori for near-field aeroacoustic source imaging," Journal of Sound and Vibration, vol. 332, no. 18, pp. 4369-4389, Fev. 2013.

[4] A. P. Young, R. Guza, P. N. Adams, W. C. O'Reilly, and R. E. Flick, "Cross-shore decay of cliff top ground motions driven by local ocean swell and infragravity waves," Journal of Geophysical Research: Oceans (1978-2012), vol. 117, no. C6, 2012.

[5] G. Birot, L. Albera, F. Wendling, and I. Merlet, "Localisation of extended brain sources from EEG/MEG: the ExSoMUSIC approach," Elsiever Neuroimage, vol. 56, no. 1, pp. 102-113, May 2011.

[6] K.I.Pedersen, P.E.Mogensen, and B.H.Fleury, "A stochastic model of the temporal and azimuthal dispersion seen at the base station in outdoor propagation environments," IEEE Transaction on Vehicular Technology, vol. 49, no. 2, Mar. 2000.

[7] A.Zoubir, Y.Wang, and P. Chargé, "Efficient subspace-based estimator for localization of multiple incoherently distributed sources," IEEE Transaction on Signal Processing, vol. 56, no. 2, pp. 532-542, Feb. 2008.

[8] A.Hu, T.Lv, H.Gao, Z.Zhang, and S.Yang, "An ESPRITbased approach for 2-d localization of incoherently distibuted sources in massive MIMO SYSTEMS," IEEE Journal of Selected Topics in Signal processing, 2014.

[9] M.Souden, "A two-stage approach to estimate the angles of arrival and the angular spreads of locally scattered sources," IEEE Transaction on Signal Processing, vol. 56, no. 5, pp. 1968-1983, May 2008.

[10] A.Zoubir and Y.Wang, "Efficient DSPE algorithm for estimating the angular parameters of coherently distributed sources," Signal Processing, vol. 88, pp. 1071-1078, 2007.

[11] A.Swindlehurst and T.Kailath, "A performance analysis of subspace method in the presence of model errors : Case of MUSIC," IEEE Transactions on Signal Processing, vol. 40, no. 7, pp. 1758-1773, July 1992.

[12] A.Kangas, P.Stoica, and T.Soderstrom, "Finite sample and modeling error effects on ESPRIT and MUSIC diretion estimators," Radar, Sonar and Navigation, IEE Proceedings, vol. 141, no. 5, pp. 249-255, Oct. 1994.

[13] A. Ferréol, P. Larzabal, and M. Viberg, "Statistical analysis of the MUSIC algorithm in the presence of modeling errors, taking into account the resolution probability," IEEE Transactions on Signal Processing, vol. 58, no. 5, pp. 4156-4166, Aug. 2010.

[14] S. Marcos, Les méthodes à haute résolution, traitement d'antenne et analyse spectrale, $\mathrm{H}$. S. Publications, Ed. 8,quai du March-Neuf,75004,Paris: Editions HERMES, 1998, no. ISSN 1159-103X.

[15] D. Astély and B. Ottersten, "The effects of local scattering on direction of arrival estimation with music," Signal Processing, IEEE Transactions on, vol. 47, no. 12, pp. 3220-3234, 1999.

[16] R. Raich, J. Goldberg, and H. Messer, "Bearing estimation for a distributed source: Modeling, inherent accuracy limitations and algorithms," Signal Processing, IEEE Transactions on, vol. 48, no. 2, pp. 429-441, 2000.

[17] W.Xiong, J.Picheral, and S.Marcos, "Performance analysis of MUSIC for spatially distributed sources," submitted to IEEE Transactions on Signal Processing. Available: http://hal-supelec.archives-ouvertes.fr/hal-01069045. 